\title{
THE DYNAMICS OF MISCIBLE FLUIDS: A SPACE FLIGHT EXPERIMENT (MIDAS)
}

\author{
T. Maxworthy, Universiy of Southern California, Los Angeles, CA \\ E. Meiburg, University of Santa Barbara, Santa Barbara, CA \\ R. Balasubramaniam and N. Rashidnia, National Center for Microgravity Research, \\ NASA Glenn Research Center, Cleveland, $\mathrm{OH}$ \\ R. Lauver, NASA Glenn Research Center, Cleveland, $\mathrm{OH}$.
}

\begin{abstract}
We propose a space flight experiment to study the dynamics of miscible interfaces. A less viscous fluid displaces one of higher viscosity within a tube. The two fluids are miscible in all proportions. An intruding "finger" forms that occupies a fraction of the tube. As time progresses diffusion at the interface combined with flow induced straining between the two fluids modifies the concentration and velocity distributions within the whole tube. Also, under such circumstances it has been proposed that the interfacial stresses could depend on the local concentration gradients (Korteweg stresses) and that the divergence of the velocity need not be zero, even though the flow is incompressible. We have obtained reasonable agreement for the tip velocity between numerical simulations (that ignored the Korteweg stress and divergence effects) and physical experiments only at high Peclet Numbers. However at moderate to low $P e$ agreement was poor. As one possibility we attributed this lack of agreement to the disregard of these effects. We propose a space experiment to measure the finger shape, tip velocity, and the velocity and concentration fields. From intercomparisons between the experiment and the calculations we can then extract values for the coefficients of the Korteweg stress terms and confirm or deny the importance of these stresses.
\end{abstract}

\section{Introduction and Background}

\section{$\underline{\text { Scientific overview }}$}

The goal of this experiment is to study the dynamics of miscible interfaces, both from a scientific and a practical point of view. We are interested in trying to unravel a

Copyright $\odot 2001$ by the American Institute of Aeronautics and Astronautics, Inc. No copyright is asserted in the United States under Title 17, U.S. Code. The U.S. Government has a royalty-free license to exercise all rights under the copyright claimed herein for Govermmental Purposes. All other rights are reserved by the copyright owner number of difficult problems that have arisen in the study of miscible interfaces. When two fluids, with different densities, are in relative motion and inter-diffuse, the resulting equations of motion contain effects that are not present in more conventional systems. Even if the two fluids are incompressible, the usual mass-averaged velocity is not divergence-free in the mixing region. However, computational simulations of such fluid flows routinely assume this effect to be small (without providing a quantitative estimate for its size), and employ a non-divergent velocity field. Obviously, this approach is open to criticism, and it is important to establish its range of validity. In addition, as discussed by Joseph and Renardy (1993), other stresses, called Korteweg stresses (Korteweg 1901) could be important in regions of large concentration gradients. Not only the magnitude of the coefficients in the expressions for these stresses, but also their sign, are presently unknown. A rough estimate is provided by Davis (1988), who puts forward scaling arguments that indicate the order of magnitude these stresses might take. Petitjeans and Maxworthy (1996) performed experiments on the displacement of a viscous fluid (glycerine) by a less viscous fluid (a glycerine/water mixture) in a capillary tube, under normal gravity. Corresponding simulations for the same fluids were carried out by Chen and Meiburg (1996) on the basis of the conventional Stokes equations, i.e., without accounting for nonsolenoidal effects or Korteweg stresses. These fluids are miscible and there is no meniscus that separates the liquids as they mix. The experiments were carried out in capillary tubes of small diameters, in order to minimize the effects of gravity. The velocity and concentration fields could not be measured in any detail in these experiments, due to the small size of the capillary tube. Therefore, the influence of the nondivergence-free velocity field and the Korteweg stresses

This is a preprint or reprint of a paper intended for presentation at a conference. Because changes may be made before formal publication, this is made available with the understanding that it will not be cited or reproduced without the permission of the author. 
could not be quantified directly. However, based on flow visualization and the measurement of tip velocity there were significant differences between the experiments and the corresponding simulations, which indicates that the divergence and/or Korteweg stress effects could play an important role in these flows, so that they need to be further investigated in a quantitative fashion. Under reduced gravity conditions, the experiments can be performed in larger tubes, and the measured velocity and concentration fields can be compared with numerical computations currently under way that account for the effects of the nonsolenoidal velocity field and the Korteweg stresses. This comparative analysis will enable us to measure the phenomenological coefficients in the expressions for the Korteweg stresses. In a nutshell, this is the motivation for the space experiment.

This research also has a practical motivation, since the improved understanding of the dynamics of multiphase porous media flows is a pre-requisite for progress in the fields of enhanced oil recovery, fixed bed regeneration, hydrology, filtration etc. (Homsy 1987, Dagan 1987). From basic stability theory (Chouke et al., 1959, Saffman and Taylor, 1958), we know that if in a porous environment the displacing fluid is less viscous than the displaced fluid, the unfavorable mobility profile leads to the well known fingering instability. The displacing fluid subsequently channels through the displaced zone, leaving behind a layer of the more viscous fluid at the walls, thereby reducing the efficiency of the displacement process. When the fluids are immiscible, the interfacial tension (or the Capillary number, which is a measure of the ratio of viscous to surface tension forces) determines the most unstable wavelength of the fingering instability as well as the dynamics of the fingers, as they evolve.

When the liquids are miscible, a meniscus between the two is absent, and the interfacial tension tends to zero. Thus, at first sight, the dynamics appear to be simpler and are determined by the relative importance of convection and diffusion. However, the comparison of our ground based experiments and corresponding numerical simulations suggests that a number of secondary effects become important and influence the dynamics. The non-vanishing divergence of the velocity field and the Korteweg stresses, discussed earlier, are two such phenomena. In addition, the diffusion tensor at the interface may be anisotropic, and may depend on the local velocity field. While it is true that a large effective interfacial tension is unlikely to exist at a miscible interface, Hu and Joseph (1991) have approached the stability problem based on the idea of such an effective interfacial tension. This approach was motivated by the observation that the form of miscible drops of one fluid moving in another is similar to that of immiscible fluids with a large interfacial tension. However, while it is easily possible to make such qualitative comparisons, it is much harder to establish them on sound quantitative footing. As a first step towards doing so, we have measured an effective interfacial tension on the order of $0.5 \mathrm{mN} / \mathrm{m}$ (Petitjeans and Maxworthy 1996). Further efforts in this direction have been undertaken by some of our collaborators (Petitjeans 1996, Petitjeans and Kurowski 1997, Kurowski and Misbah 1994). It is clear from the above discussion that miscible interfaces are rich in terms of dynamical phenomena that might influence its behavior. One output from this project would be an attempt to establish the proper form of the set of governing equations of motion for such flows. It is expected that these equations will be a modified form of the conventional Stokes equations, with additional or altered terms that allow for nonsolenoidal effects and Korteweg stresses. Under some circumstances, i.e. for some parameter regimes, we hope to be able to generate a suitable boundary condition to be used at a miscible interface, which will be compared with other approaches taken in the past, such as the effective interfacial tension employed by Hu and Joseph (1991).

\section{Brief literature survey}

The displacement of a resident fluid in a capillary tube by another fluid of different properties has historically served as a paradigm flow for studying the dynamics of interfacial phenomena and diffusive processes in the contact area between miscible fluids. In the immiscible case, the studies by Taylor (1961) as well as the corresponding calculations by Reinelt and Saffman (1985) represent major steps, cf. also the fundamental theoretical investigation by Bretherton (1961) for long bubbles. Taylor measured the amount of fluid displaced by injecting air into a horizontal capillary tube, initially filled with a viscous fluid, in order to calculate the amount of displaced fluid that was left behind on the tube wall. However, in Taylor's experiment the flow in the interior of the finger was dynamically unimportant because of the large viscosity ratios. Upon plotting the amount of fluid left behind vs. the capillary number $\mathrm{Ca}$ (where in this case $C a=V_{T} \mu / \sigma$, where $V_{T}$ is the finger tip velocity, $\sigma$ the surface tension at the interface and $\mu$ the viscosity of the displaced fluid), Taylor (1961) as well as Cox (1962) found a single curve for a number of fluids that increased at very low $\mathrm{Ca}$ as $C a^{1 / 2}$ and reached an asymptotic value of 0.60 for large $C a$. Numerical simulations by Reinelt and Saffman (1985), based on the Stokes equations, agreed very closely with these experiments. Unfortunately, no comparable experiments have been run for finite viscosity ratios.

Petitjeans and Maxworthy (1996) as well as Chen 
and Meiburg (1996) extended the above investigations to miscible fluids. In these flows, a cutoff length is set by diffusive effects rather than surface tension, so that in some sense the Peclet number $\left(P e_{T}=V_{T} d / D\right.$, where $d$ is the tube diameter and $D$ the diffusion coefficient at the interface) takes the place of $\mathrm{Ca}$. In addition, we also addressed finite viscosity ratios by varying the Atwood number $A t$, as well as the role of density differences (expressed by a further dimensionless parameter $F=[g \Delta \rho / \rho] d^{2} / \nu U$, where $\Delta \rho$ is the density difference across the interface, $g$ the acceleration of gravity, $\rho$ the density and $\nu$ the kinematic viscosity of the displaced fluid) by running experiments and simulations in vertical tubes. Interestingly, for large $P e$, large viscosity ratios and negligible density effects, the asymptotic amount of displaced fluid left behind on the tube walls ${ }^{1}$ matches the immiscible data. A fundamental difference between the immiscible and miscible cases is that the miscible flow can never become truly steady. Sooner or later, diffusion will cut of the supply of 'fresh' displacing fluid, and for long times the case of Poiseuille flow and Taylor dispersion (Taylor 1953) will be approached. However, both simulations and experiments show that for large values of $P e$, typically above $O\left(10^{3}\right)$, a quasisteady finger forms, which persists for a time of $O(P e)$ before it starts to decay. Note: Here $P e$ is defined using the maximum velocity in the Poiseuille flow ahead of the finger $\left(V_{M}\right)$ as in Petitjeans and Maxworthy (1996). Furthermore, depending on the strength of the gravitational forces, a variety of topologically different streamline patterns can be observed, among them some that leak fluid from the finger tip (a spike) and others with toroidal recirculation regions inside the finger. It should be pointed out that cylindrical tubes represent only one of the fundamental geometries. Another one of great importance is the plane, narrow gap between two plates, i.e., the classical Hele-Shaw configuration, cf. the review by Homsy (1987). Here many of the same phenomena arise, but in addition there is the issue of the length scale selection in the spanwise direction, or in the circumferential direction for the case of a localized injection. For the immiscible case, this issue was addressed by Paterson (1981) and Maxworthy (1989), cf. also the theoretical investigation by Park and Homsy (1984), as well as the computational study by Meiburg and Homsy (1988). The miscible equivalent has been investigated more recently by Joseph and Renardy (1993), Lajeunesse et al. (1997, 1998), as well as by Petitjeans, Chen, Meiburg and Maxworthy (1999) for the quarter five-spot geometry. It is clear that the selection of the dominant spanwise scale is not yet understood for miscible flows. Petitjeans (1999) has recently attempted to extract an effective surface

\footnotetext{
'This quantity is usually denoted by the sy'mbol $m=1-V_{M} / 2 V_{T}$
}

tension value for miscible flows by comparing miscible and immiscible Hele-Shaw displacements, but there are still some open questions regarding the validity of this procedure.

It needs to be pointed out that an improved understanding of the dynamics of miscible processes in such simple geometries as round tubes and plane channels is of fundamental importance, since these simple geometries form the basis for developing advanced models for the analysis of more general porous media displacements in such important areas as groundwater flows and enhanced oil recovery applications, e.g. Brady and Koch (1988).

\section{Limitations of current understanding}

From the above it is clear that at the present time the dynamics of miscible interfaces are not well understood. On the theoretical/computational side, the main problem is that the governing constitutive equations are unknown, so that it is not clear at this point on which equations to base the relevant simulation procedures. Employing the conventional Stokes equations does not lead to very good agreement with experiments, which is a strong indication that important new effects arise when the fluids are miscible that are not accounted for by these equations. While we have taken some first steps in this direction, it is clear that the accurate modeling of the dynamics of miscible interfaces taking into account these new effects, such as the nonsolenoidal velocity field in the mixing region and the influence of the Korteweg stresses, is in its infancy.

On the experimental side, the problem arises as to how to separate these novel effects appearing at miscible interfaces from other phenomena under conventional terrestrial conditions, where they tend to be adumbrated by density effects. Except for the first steps taken by Joseph and co-workers (Joseph and Renardy 1993), ourselves, and our collaborators Petitjeans and Kurowski, systematic studies of these effects are lacking.

\section{Proposed Space Experiment}

\section{Objectives of the space experiment}

The objective of the experiment is to observe the formation of a single finger of a miscible fluid that displaces a more viscous fluid, in a cylindrical tube. The two parameters that will be varied are 1). the flow rate $\left(V \mathrm{~cm}^{3} / \mathrm{s}\right)$, i.e., the maximum velocity $\left(V_{M}\right)$ of the Poiseuille flow in the more viscous fluid ahead of the finger and 2). the Atwood Number $\left[A t=\left(\mu_{2}-\mu_{1}\right) /\left(\mu_{2}+\mu_{1}\right)\right]$, that is one measure of the viscosity contrast between the two fluids. The finger shape and tip velocity will be determined and the velocity field inside and outside the finger will be mea- 
sured by particle pattern or image velocimetry (PIV). The concentration profile inside and outside the finger will be measured by interferometry. The results of the space experiment will be compared with numerical simulations in which the available parameters are varied until a close correspondence is obtained. If it turns out, as we suspect, that the observations can only be matched with the numerics if non-solenoidality and non-conventional stresses are included in the formulation then the conjecture, that such effects are important at miscible interfaces, will be confirmed. At the same time we will be able to determine the magnitude of these stresses and obtain numerical values for the coefficients that appear in the formulae for them.

\section{Description of the space experiment}

Conceptually, the experiments are quite simple. A cylindrical test chamber, that is $1 \mathrm{~cm}$ in diameter and 10 $\mathrm{cm}$ long, will be filled with a viscous liquid, to which small tracer particles will be added. The test chamber will be filled in situ. The test chamber will then be brought in contact with a flow manipulation system that displaces the viscous liquid by one of lower viscosity. A schematic of one possible form of the apparatus is shown in Figure 1. In this figure are shown the main test tube surrounded by a square tube filled with refractive-index-matching fluid. At either end are slide Valves ( $A$ and $B$ ) that are used to isolate the tube during filling. Piston $A$, of diameter $1 \mathrm{~cm}$, is co-axial with the tube and is used to displace fluid during cleaning operations and fill the tube with High Viscosity fluid (HVF). Piston B, diameter $0.71 \mathrm{~cm}$, is used to fill the interior of Piston $A$ with Low Viscosity fluid (LVF) and then inject it into the HVF in the main cylinder. Also show'n are the drive mechanisms for the displacements of Pistons $A$ and $B$. The drive for Piston B will be connected to the end of Piston A. The amount of LVF to be injected is approximately $4 \mathrm{ml}$ at rates between $1.2 \times 10^{-4}$ and $1.2 \times 10^{-2} \mathrm{ml} / \mathrm{s}$ ).

The displacing liquid will also contain tracer particles. The piston displacement velocity $\left(V_{P}\right)$ will be in the range 3 to 300 micron $/ \mathrm{s}$. Note that for the piston diameter chosen $(0.707 \mathrm{~cm}) V_{M}=V_{P}$. The viscosity of the displacing fluids used will be less than $1000 \mathrm{cp}$, such that the Atwood number is in the range 0.818 to 0.998 . The fluids used will be silicone oils (Dow DC-200 or Rhone-Poulenc Rhodorsil 47 series).

The displacement process will be viewed by a video camera, from one side of the test chamber. To minimize optical distortion, the refractive index of the walls of the test chamber must be as close as possible to that of the fluids. Also, the cylindrical test chamber must be enclosed inside a square chamber, and the intervening space filled with a transparent material that is also matched in the refractive index. Alternatively, the test chamber can be constructed by forming a cylindrical hole in a glass ingot of square cross-section and then grinding and polishing the inside and outside surfaces to the required accuracy.

While the quasi-steady-state flow field is probably of greatest interest the transient state can also yield interesting information. As a result the experiment is designed to look at finger evolution from the beginning of the motion until the quasi-steady state, in the moving reference frame, is well established. This distance of travel is expected to be from 8 to 9 tube diameters. The concentration field will be determined by optical interferometry and the velocity field by particle image velocimetry. In the latter case the test chamber will be illuminated by a light sheet that spans a radial plane. Under most circumstances the imaged field will be approximately $1 \times 1.5 \mathrm{~cm}$. However it is necessary to be able to focus on regions both smaller and larger than this, i.e., a zoom lens will be used. This is done in order 1). to be able to observe the details of the sharp velocity gradients that occupy, at most, regions of the order of 2 $\mathrm{mm}$ in extent and are mostly off the center axis of the tube and 2). to be able to observe the large scale motion of order $3 \mathrm{~cm}$ in axial extent in order to find the velocity of the finger (see below) and view the evolution of the finger from the beginning of its motion. Thus, in order to be able to observe the finger, at various magnifications and from two orthogonal directions, over an axial distance of about $9 \mathrm{~cm}$ and a radial distance of about $0.3 \mathrm{~cm}$ from the tube center line it will be necessary to move the tube with respect to the optical system. This means that the fluid system will be mounted on a 3D traverse driven, for example, by lead screws and pulse motors with planetary gear reductions.

An accurately constructed grid, placed inside the tube containing the oil to be displaced, will be used to calibrate the optical system before the flight. The experiments will then consist of recording two images of the pattern of the tracer particles, at a known time difference, and then using a well-documented digital pattern correlation technique to find the velocities on a regular grid.

As the fluids diffuse into each other, the concentration of each fluid will be non-uniform. The concentration gradient will especially be sharp near the finger tip. We will measure the concentration field around the finger and in the displaced fluid, by an interferometric system. The fringe shifts caused by the intruding finger will be measured and the line of sight refractive index distribution will be found. Ground based experiments 
being performed at NASA GRC show that a shearing interferometer with a Wollaston prism is well suited for this experiment. Using the known refractive index versus concentration relationship for the fluids, the radial concentration distribution can be obtained by inversion of the Abel integral, and can then be compared with numerically predicted distributions. An attractive alternative is to numerically predict the fringe pattern, and compare it directly with the one obtained experimentally.

Ground based experiments have been performed at NASA Glenn RC to visualize the concentration profile around a finger, using a common path shearing interferometer with a Wollaston prism, operating in the finite fringe mode. This interferometer measures the index of refraction gradient in a certain direction, perpendicular to the line of sight of the optical system. This direction is adjustable by rotation of the prism about the line of sight. A collimated beam of polarized light from a He-Ne laser is passed through the test cell. The light is then focused by a lens (175 $\mathrm{mm}$ focal length, $51 \mathrm{~mm}$ diameter) on a Wollaston prism, with a 20 minute divergence angle. An analyzer is placed after the prism, and the image is captured by a CCD camera, equipped with a zoom lens $(65-200 \mathrm{~mm})$.

\section{Experimental Plan}

\section{Summary of the flight experiment procedure}

The test chamber is filled with the desired fluid (1000 cs silicone oil, to which 10 micron particles are added). The pumping system is filled with the silicone oil of the desired lower viscosity also with particles suspended within it. The displacement piston will then be started at a predetermined velocity. The region to be imaged will be placed in the field of view of the camera. When the finger enters the chosen imaging location, the velocity and concentration measurement systems will be activated, and the velocity and concentration fields will be measured several times as the finger traverses the field of view. The velocity field measurement basically consists of capturing two images of the particle pattern, taken at a predetermined time apart, and the sequence is repeated at predetermined intervals until the flow state is no longer of interest. Any' particular experiment will be considered finished when the received/analyzed data shows no more interesting features, e.g., when diffusion from the quasi-cylindrical sides of the intrusion has created an interface thickness that exceeds a preset value beyond which we expect it to have no significant dynamical effect. Simultaneously with the velocity field measurements, the concentration measurement system will measure the interferometric fringes that represent the varying index of refraction field around the finger, that arises from the diffusion of the fluids. The test chamber is then ready to be cleaned and filled for the next run. The experiments will be repeated at various displacement speeds for a given fluid pair, and for different fluid pairs to span the test matrix chosen.

\section{Test matrix}

Silicone oils are the most likely candidates for the fluids. We will use 5 fluid pairs to vary $A t$ in the range $0.818<A t<0.998$. The displaced fluid will have a kinematic viscosity of $1000 \mathrm{cs}$. The kinematic viscosity of the displacing fluid will be in the range 1 to 100 cs [e.g., 1 cs $(A t=0.998) ; 3 c s(A t=0.994) ; 10 \mathrm{cs}$ $(A t=0.980) ; 50 \mathrm{cs}(A t=0.905) ; 100 \mathrm{cs}(A t=0.818)]$. For each fluid pair, the experiment will be performed at approximately 8 different displacement speeds in the range 3 - $300 \mathrm{micron} / \mathrm{s}$. A possible experimental operating region is sketched in Figure 2 where we have inserted our best guess for the line $m=1 / 2$ from existing results. We plan to span the region in $A t-P e$ space from where spike formation first starts to well into the region where a wellformed nose exists. The actual number of experiments will depend to a large extent on how much fluid can be stored within the allotted space in the International Space Station (ISS). Note: each experiment requires about 10 $\mathrm{ml}$ of HVF and slightly more than $4 \mathrm{ml}$ of LVF! A few experiments will be run twice in order to test the repeatability and accuracy of the system

\section{Experimental Requirements}

\section{Summary of experimental requirements}

In summary the following equipment is needed to carry out the experimental program: An experimental chamber and flow manipulating hardware. The latter including two slide valves operated by solenoids; two fluid displacement pistons driven by geared pulse motors; six pressurized piston-type fluid holding tanks with mixers; a small high pressure air supply and control valve; one bladder or piston-type dump tank. Carriage to traverse the apparatus within the field of view of the camera(s). Electronics and computers to control sequencing of the various components including the data acquisition system and apparatus traverse. Data acquisition system: Wollaston prism based interferometer system; light source for PIV; high resolution (1024×1024 pixels) digital video camera(s) with 6 $7 \times$ zoom lens(es); power supplies; computer and up/down data link. Programmable data acquisition, i.e., an ability to change experimental conditions as the experimental sequence progresses. Real time data storage within the ISS. Close to real time ground-based data reception/analysis. 


\section{Experimental chamber}

The experiment will be conducted in a transparent cylindrical tube that has an inner diameter of $10 \mathrm{~mm}$, and a length of $100 \mathrm{~mm}$. The outer walls of the tube must be square. One option is to form a cylindrical hole in a glass ingot and then grind and polish to the required accuracy. Another possibility is to surround the cylindrical glass tube by a square one, and fill the intervening space with a suitable liquid that has the same refractive index as the glass of the tubes. The latter possibility is shown in Figure 1. The exterior walls of the test chamber must be optically flat to within $1 / 4$ wavelength of the light used, so that interferometric measurements can be performed without distortion.

\section{Filling and control of the fluids in the test chamber}

The displacement velocity of the fluids must be controlled to within $1 \%$ of the mean or average velocity. The average displacement velocity will be in the range 3 to $300 \mathrm{micron} / \mathrm{s}$ (estimated flow rates of $1.2 \times 10^{-4}$ to $1.2 \times 10^{-2} \mathrm{~cm}^{3} / \mathrm{s}$ ). The apparatus shown in Figure 1 operates in the following way:

The experimental sequence starts with pistons A and $B$ resting at stations $I$ and the end of Piston $A$ respectively, with valve $A$ closed and $B$ open.

Pistons $\mathrm{A}$ and $\mathrm{B}$ are withdrawn together to station 2 bringing with it HVF from a pressurized container. Valve $B$ is then closed.

Piston $\mathrm{B}$ is withdrawn for a distance of approximately $10 \mathrm{~cm}$ bringing with it LVF from one of six pressurized containers each holding a fluid of different viscosity.

Valve $B$ is opened. Piston $A$ is moved forward to station 3 in the view of the camera. Some of the HVF is returned to its reservoir. Valve $A$ is opened. Piston $B$ is moved at an accurately known speed to empty its cylinder, LVF enters the main chamber and an experiment begins.

At the end of an experiment Pistons $\mathrm{A}$ and $\mathrm{B}$ are displaced to station $I$ and the mixture of HVF and LVF dumped into the holding tank

The sequence is then repeated as many times as required within the constraints of the fluid supply.

In this way the experiment can be automated and only minute amounts of the two fluids will come into contact to cause sample contamination except during an actual experiment.

\section{Diagnostics and imaging requirements}

The formation of the finger will be observed from the moment the displacement process begins. Measurements of the early evolution, as the finger forms, are of interest and will be taken. The evolution of the finger will then be followed with the whole apparatus being moved to the appropriate location on a computer controlled carriage so that the part of the finger of interest will always be in the field of view of the fixed optical system. Measurements will be performed to obtain the unsteady and quasi-steady velocity and concentration fields inside and outside the finger. Detailed measurements will be made of the velocity and concentration fields at the interface between the two fluids.

Field of view:

The field of view for the camera observing the formation of the finger is to be variable and depends on the question to be answered. Thus, for most tests it will be $1 \times 1.5 \mathrm{~cm}$, i.e., spans the diameter of the tube. When the field in the neighborhood of the interface is to be studied it will be of the order of $3 \times 4.5 \mathrm{~mm}$ and when the field of the whole ogival portion of the nose is to be studied it will be $2 \times 3 \mathrm{~cm}$, i.e., a total zoom ratio of six to seven. The location of the field of view for the velocity field measurement system depends, as above, on the question under consideration and the location of the tip of the finger. The velocity field measurements will be made when the finger-tip location is between zero and nine tube diameters from the starting end of the test chamber. The field of view and location for the concentration field measurements will probably be the same. To check that the evolution of the finger is axisymmetric, it would be preferable to measure the concentration field from a direction that is at an angle of $90^{\circ}$ to that for the measurement of the velocity field. This probably means that two different cameras will be needed to view the two different fields. Details depend on the possibly conflicting requirements of the two systems and whether or not the system will be incorporated into the French DECLIC instrument package.

Particle pattern (image) velocimetry $[\mathrm{PP}(\mathrm{I}) \mathrm{V}]$ :

A light sheet approximately $0.7 \mathrm{~mm}$ thick spanning the center of the tube will illuminate 10 micron diameter hollow glass spheres at a concentration of about $500 / \mathrm{mm}^{3}$. i.e., approximately $1.7 \times 10^{7}$ total or a volume fraction of less than $1 \%$. Two digital images of those particles in the field of view of the $1024 \times 1024$ 
pixel digital progressive scan video camera will be taken at a time interval that depends upon the flow rate under consideration and the magnification being used, i.e., which of the three regions of the flow is being studied. Ideally one wants a particle pattern displacement between images of about 5 to 10 pixels. Since the dynamic range of the velocity field is substantial it may be necessary to study the same field at different inter-frame times in order to improve the accuracy of the measurements. These pairs of images will be taken at intervals that are sufficient to reveal 1). the unsteady evolution of the finger and 2). the ultimate quasi-steady state.

\section{Concentration measurement system:}

Measurement of the gradient of the index of refraction around the finger, by a shearing interferometer using a Wollaston prism, is well suited for this experiment. The light source will be a He-Ne laser ( 632.8 nanometer wavelength). The divergence angle of the Wollaston prism will be 20 minutes, and the focal length of the focusing lens will be $175 \mathrm{~mm}$. This yields a sheared beam separation distance of $1.018 \mathrm{~mm}$. The interferometer will be used in the finite fringe mode, and the fringe shifts caused by the index of refraction gradient in the test cell will be determined. The field of view for the interferometer will range from $10 \times 15 \mathrm{~mm}$ to $3 \times 4.5 \mathrm{~mm}$. The former will cover the cross section of the tube, and the latter is necessary to obtain measurements near the mixing front. A spatial resolution of 10 micron is required. A $1024 \times 1024$ pixel digital progressive scan video camera is adequate for the measurements. There is a possibility of interference between the two optical systems, i.e., the particles may adversely affect with the operation af the interferometer. Experiments will be carried out at NASA Glenn RC to test for this possibility.

\section{Temperature and pressure measurement}

The temperature of the fluids should be measured (accuracy $0.02^{\circ} \mathrm{C}$ ) and controlled such that it is uniform to within $\pm 0.1^{\circ} \mathrm{C}$ of its nominal value $20^{\circ} \mathrm{C}$. The differential expansion of the two fluids can give an erroneous velocity reading if a larger temperature change than $0.10^{\circ} \mathrm{C}$ takes place over a short period of time. Also, the temperature of the fluids influences the viscosity and hence the Atwood number. The pressure in the test cell must be close to the ambient pressure and constant.

\section{Acceleration environment}

The quoted average absolute steady state D C "g" level in the Space Shuttle/Space Station, of $2 \times 10^{-6} g_{0}$, is more than sufficient for the proposed experiments. Based on the analysis given in section 3.1 it is unlikely that moderate to high frequency deviations from the mean level with less than an absolute magnitude of $10^{-4} g_{0}$ will have a significant effect on the dynamics of interest and this is well within the range measured.

\section{References}

Brady, J.F. and Koch, D.L. 1988 in Disorder and Mixing. edited by E.Guyon et al., NATO ASI Series E, Academic Press, New York.

Bretherton, F.P. 1961 The motion of long bubbles in tubes. J. Fluid Mech. 10, 166.

Chen, C.-Y. and Meiburg, E. 1996 Miscible displacements in a capillary tube.Part 2 . Numerical simulations. J. Fluid Mech. 326, 57.

Chen, C.-Y. and Meiburg, E. 1998a Miscible porous media displacements in the quarter five-spot configuration. Part 1: The homogeneous case. J. Fluid Mech. 37 I, 233.

Chen, C.-Y. and Meiburg, E. $1998 \mathrm{~b}$ Miscible porous media displacements in the quarter five-spot configuration. Part 2: Effect of heterogeneities. J. Fluid Mech. $371,269$.

Chouke, R.L., Meurs, P. van and Pol, C. van der 1959 The instability of slow, immiscible, viscous liquidliquid displacements in permeable media. Trans. AMME $216,188$.

Clift, R., Grace, J. and Weber, M. 1978 Bubbles, Drops and Particles. Academic Press, New York.

Cox, B.G. 1962 On driving a viscous fluid out of a tube. J. Fluid Mech. 14, 81 .

Dagan, G. 1987 Theory of solute transport in groundwater. Ann. Rev. Fluid Mech. 19, 183.

Davis, H.T. 1988 A theory of tension at a miscible displacement front. In Numerical Simulation in Oil Recovery, IMA Volumes in Mathematics and Its Applications II, ed. M. Wheeler, Springer, Berlin.

Homsy, G.M. 1987 Viscous fingering in porous media. Ann. Rev. Fluid Mech.19, 271.

Hu, H. and Joseph, D.D. 1991 Interfacial tension between miscible liquids. Army High Performance Computing Research Centre, University of Minnesota, preprint 91-58. 
Joseph, D.D. and Renardy, Y.Y. 1993 Fundamentals of two-fluid dynamics.Part II. Lubricated transport, drops, and miscible liquids. Springer series on Interdisciplinary Applied Mathematics, Springer, New York.

Korteweg, D. 1901 Sur la forme que prennent les equations du mouvement des fluides si lon tient compte des forces capillaires causees par des variationsde densite. Arch. Neerl. Sci. Ex. Nat., Series II 6, 1.

Kurowski, P. and Misbah, C. 1994 A non-standard effect of diffusion on a fictitious front between miscible fluids. Eur. Phys. Letts. 29, 309

Lajeunesse, E., Martin, J., Rakotomalala, N., Salin, D. 1997 3D instability of miscible displacements in a Hele-Shaw cell. Phys. Rev. Lett. 79, 5254.

Lajeunesse, E., Martin, J., Rakotomalala, N., Salin, D., and Yortsos, Y.C. 1998 Miscible displacement in a Hele-Shaw cell at high rates. J. Fluid Mechs., 398, 299.

Maxworthy, T. 1989 Experimental study of interface instability in a Hele-Shaw cell. Phys. Rev. A 39, 5863.

Meiburg, E. and Homsy, G.M. 1988 Nonlinear, unstable, viscous fingers in Hele-Shaw flows. Part 2: Numerical simulations. Phys. Fluids 31, 429.

Park, C-W. and Homsy, G.M., 1984, Two-phase displacement in Hele Shaw cells, J. Fluid Mechs., 139, 291.

Paterson, L. 1981 Radial fingering in a Hele-Shaw cell. J. Fluid Mech. 113, 513.

Petitjeans, P. 1996 Une tension de surface pour les fluides miscibles. C.R. Acad. Sci. Paris, Serie Irb, 322, 673.

Petitjeans, P. 1999 Gravitational instability between miscible fluids. Bull. Am. Phys. Soc. 44, 122.

Petitjeans, P. and Maxworthy, T. 1996 Miscible displacements in a capillary tube. Part 1. Experiments. J. Fluid Mech. 326, 37.

Petitjeans, P. and Kurowski, P. 1997 Fluides non miscibles/fluides miscibles: des similitudes interessantes. C.R. Acad. Sci. Paris, Serie IIb, 325, 587.

Petitjeans, P., Chen, C.-Y., Meiburg, E. and Maxworthy, T. 1999. Miscible 1/4-Five Spot Displacement in a Hele Shaw cell and the Role of Flow-induced
Dispersion. Phys. Fluids., 11, no. 7, 1705.

Reinelt, D.A. and Saffman, P.G. 1985 The penetration of a finger into a viscous fluid in a channel and tube, SIAM J. Sci. Stat. Comput. 6, 542.

Saffman and Taylor 1958 The penetration of a finger into a porous medium in a Hele Shaw cell containing a more viscous fluid, Proc. Roy. Soc. A245, 312.

Taylor, G.I. 1953 Dispersion of soluble matter in solvent flowing slowly through a tube. Proc. Roy. Soc. A $219,186$.

Taylor, G.I. 1961 Deposition of a viscous fluid on the wall of a tube. J. Fluid Mech. 10, 161.

Zimmerman, W.B. and Homsy, G.M. 1991 Nonlinear viscous fingering immiscible displacements with anisotropic dispersion. Phys. Fluids A 3, 1859. 


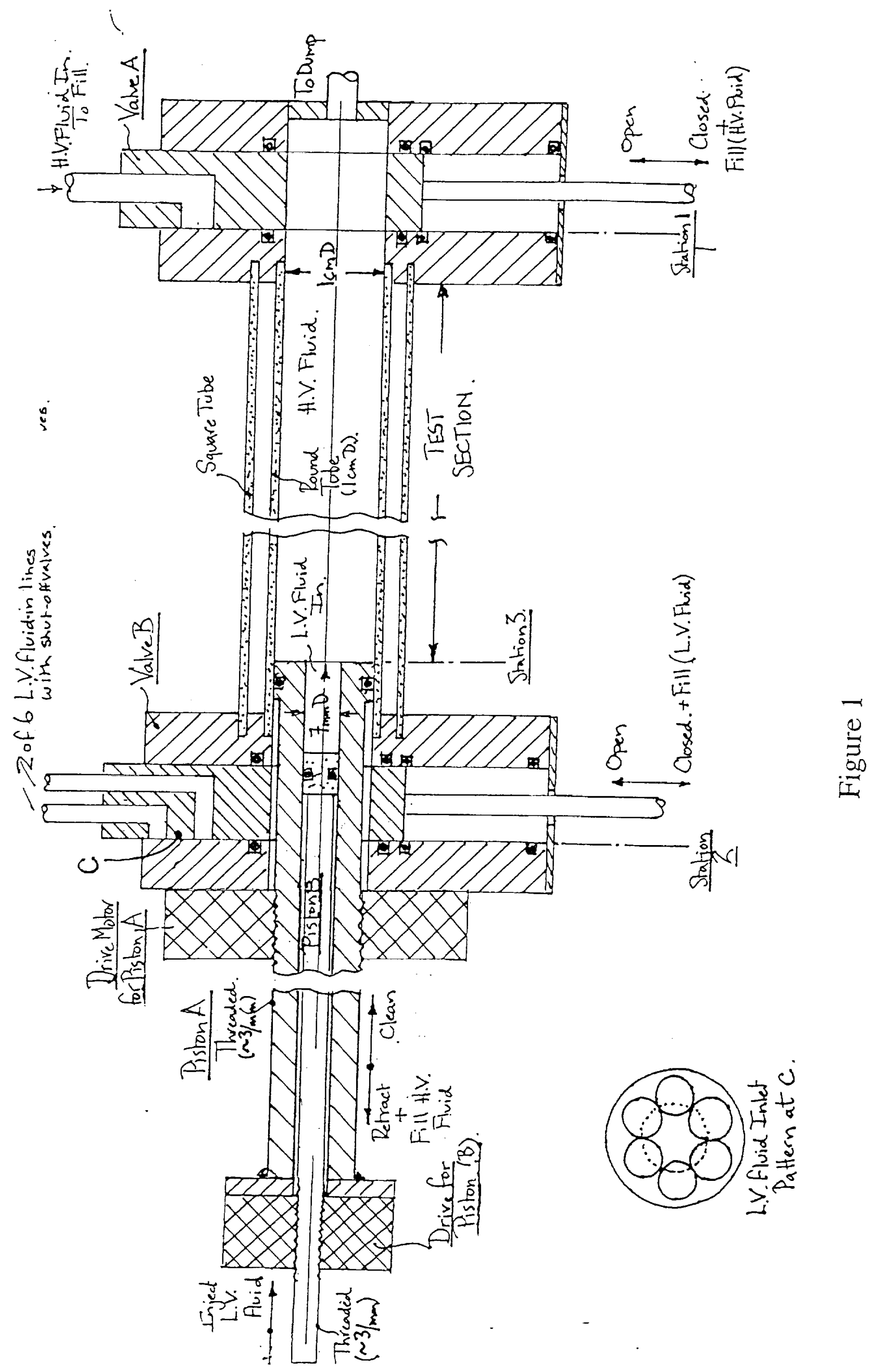




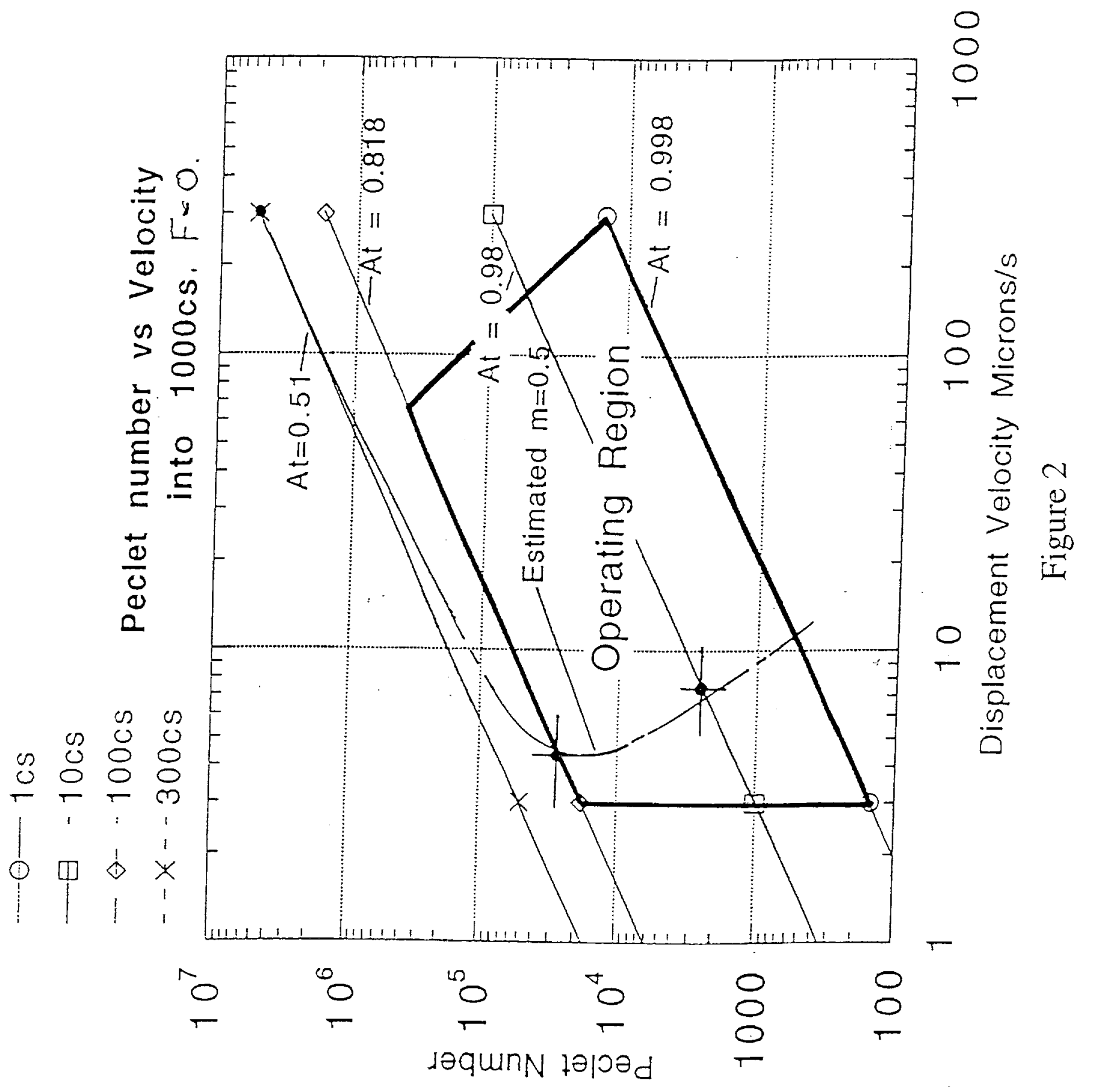

